\title{
Improvement of Gain and Elevation Tilt Angle Using Metamaterial Loading for Millimeter-Wave Applications
}

\author{
Abdolmehdi Dadgarpour, Student Member, IEEE, Behnam Zarghooni, Student Member, IEEE, \\ Bal S. Virdee, Senior Member, IEEE, and Tayeb A. Denidni, Senior Member, IEEE
}

\begin{abstract}
Elevation-plane beam tilting is demonstrated for a printed dipole antenna operating over 57-64 GHz. This is achieved using a $3 \times 4$ array of high refractive-index metamaterial (HRIM) unit cells. The unit cell comprises a modified $\mathbf{H}$-shaped structure with stub loading to control the refractive index of the unit cell over a finite frequency range. Integration of the $3 \times 4$ array in the H-plane of a dipole antenna is shown to deflect the main beam by $+30^{\circ}$ with respect to the endfire direction over 57-64 GHz. In addition, the proposed technique provides $5 \mathrm{~dB}$ gain enhancement
\end{abstract}

Index Terms-Beam switching, beam tilting, beamforming network, dipole antennas, metamaterials, millimeter waves.

\section{INTRODUCTION}

C OMMUNICATION in the unlicensed $60-\mathrm{GHz}$ band $(57-66 \mathrm{GHz})$ has attracted great attention for short-range multi-Gb/s data rate applications such as high-definition video streaming using the IEEE 802.11ad WiGig standard. One distinguishing feature of the $60-\mathrm{GHz}$ communication is its high propagation loss due to the extremely high carrier frequency and the oxygen absorption peaks at this frequency band. To combat this, directional antenna with high directivity gain can be adopted to obtain sufficient link budget. This can be accomplished using antenna arrays that provide advantages of interference mitigation and multipath suppression [1]. The challenge with directional antennas is beam alignment between the communication pair.

Various techniques have been proposed to date for steering the main beam of antennas including integrated lens antennas at millimeter wave [2], [3]. The drawback of this approach is its bulky framework. In [4], an array of dipole antenna is mounted perpendicular to an electromagnetic band-gap (EBG) groundplane to realize beam tilting for mobile base stations. With this technique, the beam tilting angle is limited to $25^{\circ}$. The physical size of the antenna at $3.5 \mathrm{GHz}$ is $2.6 \lambda \times 0.63 \lambda \times 0.84 \lambda$. Comb-line antenna array in [5] provides a tilt angle of $30^{\circ}$ at

Manuscript received March 28, 2015; accepted June 06, 2015. Date of publication June 23, 2015; date of current version February 26, 2016.

A. Dadgarpour, B. Zarghooni, and T. A. Denidni are with the Institut National de la Recherche Scientifique (INRS-EMT), University of Quebec, Montreal, QC H5A 1K6, Canada (e-mail: abdolmehdi.dadgarpour@emt.inrs.ca; zarghooni@emt.inrs.ca; denidni@emt.inrs.ca).

B. S. Virdee is with the Center for Communications Technology, London Metropolitan University, London N7 8DB, U.K. (e-mail: b.virdee@londonmet. ac.uk).

Color versions of one or more of the figures in this letter are available online at http://ieeexplore.ieee.org.

Digital Object Identifier 10.1109/LAWP.2015.2449235
$76 \mathrm{GHz}$. This antenna is composed of several rectangular radiating elements directly connected to a straight feeding microstrip line. The radiating elements are inclined $45^{\circ}$ from the feeding line. The drawback of this configuration is its large physical size, i.e., $15.2 \lambda \times 2.5 \lambda \times 0.03 \lambda$, as it requires two lines of 26-element linear array and its sidelobe is $-5 \mathrm{~dB}$. Authors in [6] have demonstrated the beam-tilting phenomenon can be realized using high refractive-index metamaterial loading that is integrated within the antenna structure.

With this technique, the beam tilt angle is limited to $17^{\circ}$ in the azimuth plane at $\mathrm{C}$-band. In addition, over its operating frequency range, the gain of the antenna is restricted to $1.1-2.4 \mathrm{~dB}$.

This letter presents results on 1-D antenna beam deflection technique over $57-64 \mathrm{GHz}$ using a novel $3 \times 4$ array of high refractive-index metamaterial (HRIM) unit cells. The antenna is capable of deflecting the main beam by $+30^{\circ}$ with respect to the endfire direction, and the deflection is accompanied by peak gain of $12 \mathrm{dBi}$ at $63 \mathrm{GHz}$.

\section{Mechanism of BeAm-Tilting}

It has been shown in [2] that placing a dielectric hemispherical lens over an antenna results in beam deflection. The angle of deflection can be controlled by using lenses of different radii. In this letter, we have realized beam deflection in the elevation plane of a planar dipole antenna by loading it with an array of HRIM unit cells. The metamaterial array is loaded directly onto the dipole antenna in the elevation-plane in the path of the radiation. The array effectively acts like a hemispherical lens that deflects the radiation beam. Moreover, the array effectively increases the antenna aperture to enhance its gain performance.

Beam tilt angle can be determined from the geometry of the HRIM loaded dipole antenna, shown in Fig. 1, where there is phase difference between the paths of lengths " $a$ " along the substrate surface, which guides a TE surface wave and the freespace path of length $\sqrt{\left(L^{2}+a^{2}\right)}$, where $a$ is the distance of dipole antenna to the HRIM region, and $L$ is the length of HRIM region in the $z$-direction.

In order to implement the proposed technique, the metamaterial unit-cell structure, shown in Fig. 2, is employed. This is a modified version of the $\mathrm{H}$-shaped meandered-line metamaterial structure in [6]. The unit cell is constructed on a Rogers RT5880 substrate with the thickness $(h)$ of $0.508 \mathrm{~mm}$, permittivity $\left(\varepsilon_{\mathrm{r}}\right)$ of 2.3 , and loss tangent of 0.0009 .

The unit cell's effective permittivity, permeability as well as refractive-index were extracted by modeling the structure on Ansoft HFSS, where the PEC and PMC boundary conditions were applied along the $y z$ - and $x z$-planes, and the two ports 


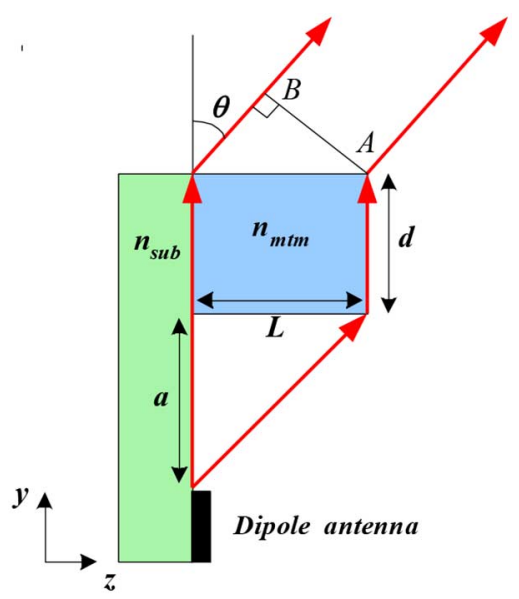

Fig. 1. Mechanism of beam tilting in the H-plane of the dipole antenna.

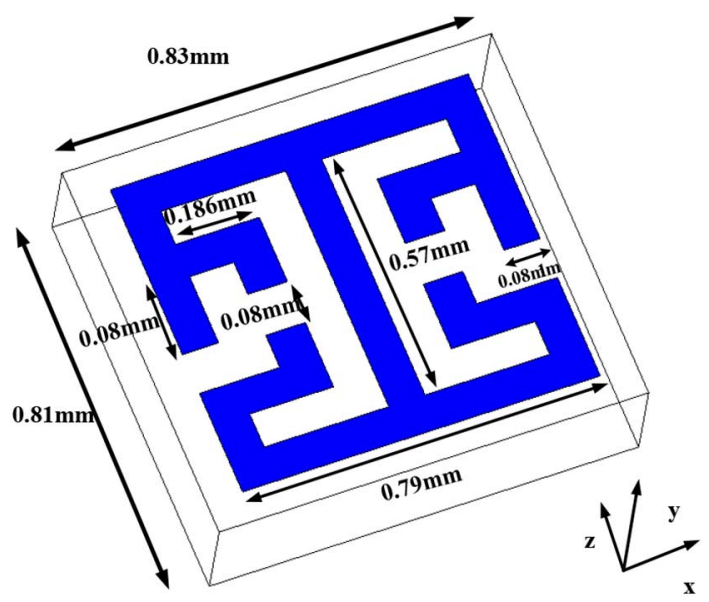

Fig. 2. Geometry of the proposed HRIM unit cell implemented on a dielectric substrate.

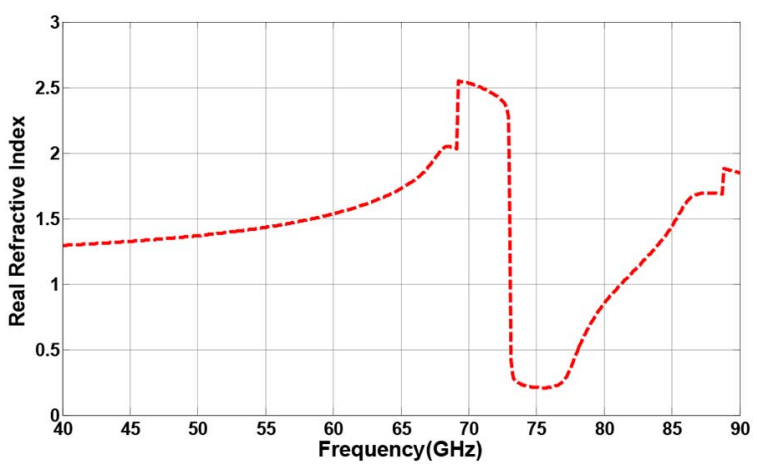

Fig. 3. Real part of effective refractive index of the HRIM unit cell.

were located in $x$-direction. $S$-parameters of the unit-cell structure and its characterizing parameters were extracted using the algorithm in [7]. Fig. 3 shows the magnitude of the effective refractive index of the proposed unit cell is between 1.46 and 1.67 over 57-64 GHz, which is larger than effective refractive index of antenna substrate (i.e., 1.28).

\section{Beam Deflection of a Single-Dipole Antenna}

In this section, the characteristics of a single-dipole antenna are investigated when it is loaded with a $3 \times 4$ array of HRIM unit cells in the elevation plane $(y z)$, as shown in Fig. 4. The dipole antenna was constructed on Rogers RT5870 substrate

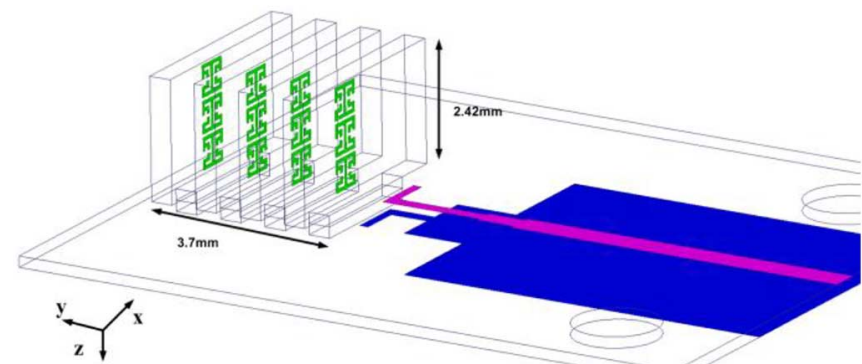

Fig. 4. Configuration of the antenna embedded with a $3 \times 4$ array of the proposed HRIM unit cells on upper surface of antenna substrate.

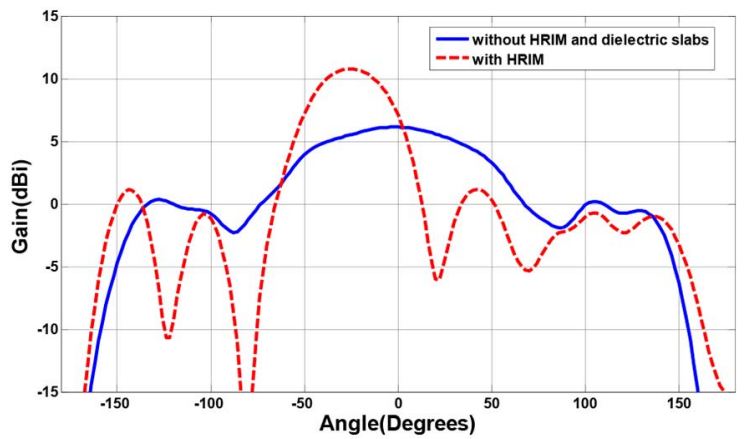

Fig. 5. Radiation patterns of proposed antenna in the H-plane $(y z)$ with proposed unit cells; and a conventional dipole antenna with no HRIM loading at $60 \mathrm{GHz}$.

with thickness of $0.254 \mathrm{~mm}$ and permittivity of 2.3 . The index of refraction plotted in Fig. 3 is applicable to an infinite volume of HRIM unit cells. However, what has been built is only a small slice, or a limited volume, of the array of slabs shown in Fig. 4. Most of the volume of this array of slabs has a lower index of refraction, and it is described by: $\left[\left(\varepsilon_{\mathrm{r} 1} t_{1}+\varepsilon_{\mathrm{r} 2} t_{2}\right)\left(t_{1}+t_{2}\right)\right.$, where $\varepsilon_{\mathrm{r} 1}\left(\varepsilon_{\mathrm{r} 2}\right)$ is the relative permittivity of the dielectric slabs (of the air region) and $t_{1}\left(t_{2}\right)$ is the thickness of the slab region (air region). As constructed, the HRIM metamaterial is really an array of resonant scatterers that lie in a plane (the H-plane) as opposed to occupying the entire volume of the region associated with the slabs.

The radiation patterns of the antenna with and without unit cell loading in the H-plane ( $y z$ ) of the antenna is shown in Fig. 5. The results show the direction of the main beam in the H-plane tilts by $-30^{\circ}$ with respected to the endfire direction. In addition, this deflection is accompanied by $5 \mathrm{~dB}$ gain enhancement compared to dipole antenna with no HRIM loading. This indicates the HRIM loading essentially behaves as a meta-lens that effectively increases the aperture size of antenna yielding antenna gain enhancement.

\section{EXPERIMENTAL RESULTS}

A photograph of the fabricated single-dipole antenna with a $3 \times 4$ array of metamaterial unit cells is shown in Fig. 6 . The HRIM unit cells were constructed on a Rogers RT5880 dielectric slab with thickness of 0.508 , and the four slabs are integrated in the H-plane of the antenna. A 1.85-mm end-launch Southwest Microwave connector was used in the antenna measurements.

The measured reflection coefficient of the antenna, shown in Fig. 7, is less than $-15 \mathrm{~dB}$ between $55-65 \mathrm{GHz}$. The simulated and measured radiation patterns of the dipole antenna with HRIM loading at 58,60, and $62 \mathrm{GHz}$ are shown in Fig. 8. The 


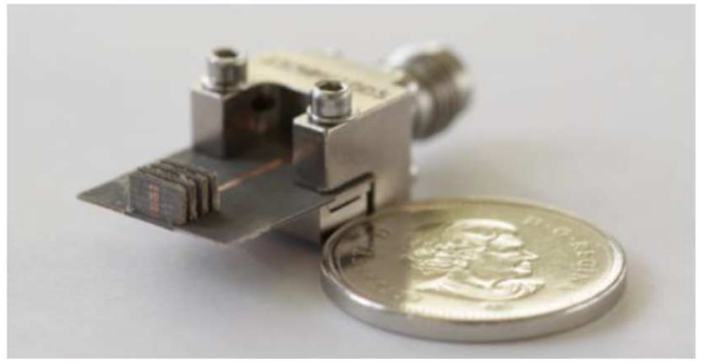

Fig. 6. Photograph of the single-feed antenna with HRIM loading in elevation plane.

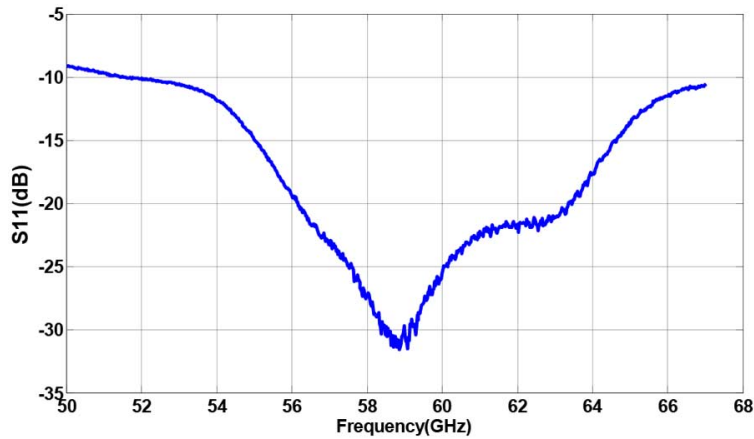

Fig. 7. Measured reflection coefficient of the single-dipole antenna with HRIM unit-cell loading.

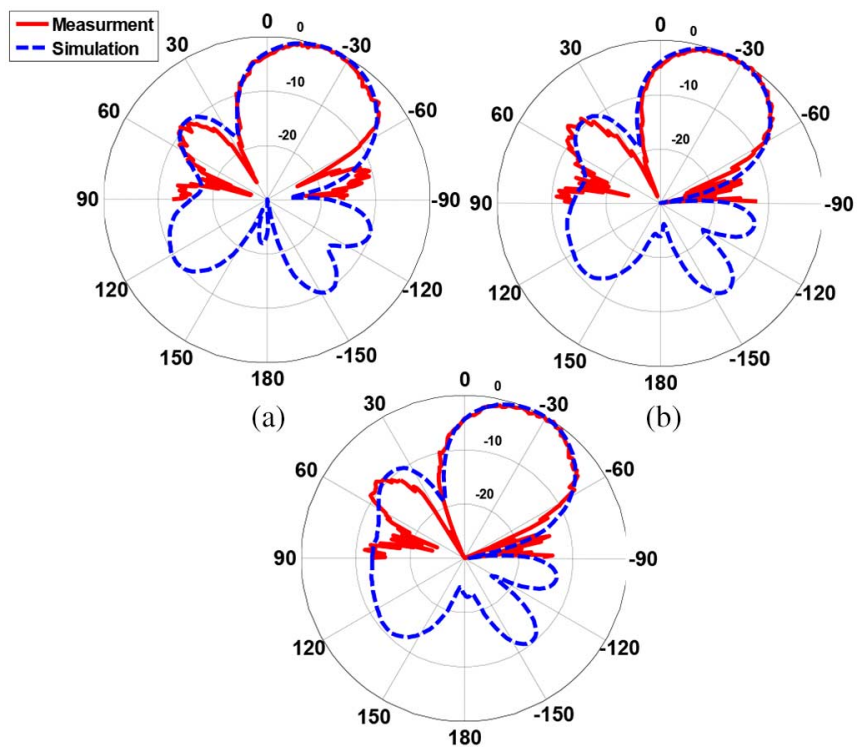

(c)

Fig. 8. Normalized radiation patterns of the single-dipole antenna with $3 \times 4$ HRIM in the H-plane (yz) at (a) 58, (b) 60 , and (c) $62 \mathrm{GHz}$.

measured results confirm the main beam of the antenna tilts by $30^{\circ}$.

The radiation pattern in the E-plane at $60 \mathrm{GHz}$, shown in Fig. 9, remains virtually unaffected and is directed toward the endfire direction. The measured gain of the antenna at a beam tilt angle of $+30^{\circ}$, shown in Fig. 10, is greater than $10 \mathrm{dBi}$ over 57-64 GHz. The gain peaks to $12 \mathrm{dBi}$ at $63 \mathrm{GHz}$.

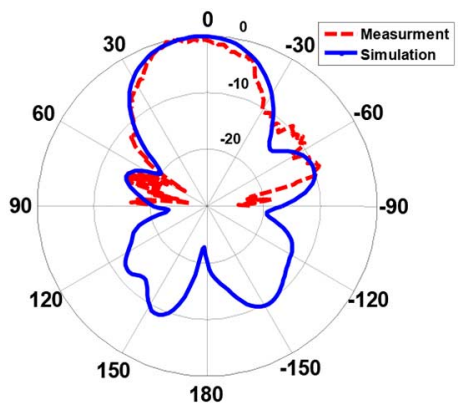

Fig. 9. Normalized radiation pattern of the single-dipole antenna with $3 \times 4$ array of HRIM unit cells in the E-plane $(x y)$ at $60 \mathrm{GHz}$.

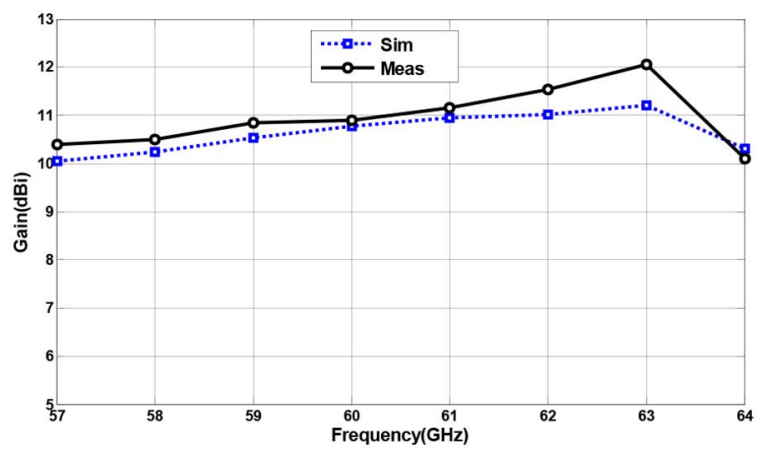

Fig. 10. Measured and simulated antenna gain.

\section{CONCLUSION}

High refractive-index metamaterial HRIM unit cells are shown to tilt the direction of a dipole antenna's main beam by a prescribed angle. This was accomplished by using an array of HRIM that were directly embedded in the elevation plane of the planar antenna. Measured results confirm the direction of the antenna's main beam can be deflected by $+30^{\circ}$ in the H-plane (elevation plane) with a $3 \times 4$ array of HRIM. With the proposed technique, the antenna exhibits a peak gain of $12 \mathrm{dBi}$ at $63 \mathrm{GHz}$ when the beam was tilted by $+30^{\circ}$. This constitutes gain enhancement of $5 \mathrm{dBi}$ compared to the unloaded state.

\section{REFERENCES}

[1] K. Hosoya et al., "Multiple sector ID capture (MIDC): A nove beamforming technique for $60 \mathrm{GHz}$ band multi-Gbps WLAN/PAN systems," IEEE Trans. Antennas Propag., vol. 63, no. 1, pp. 81-96, Dec. 2014

[2] A. Artemenko, A. Maltsev, R. Maslennikov, A. Sevastyanov, and V. Ssorin, "Beam steerable quartz integrated lens antenna for $60 \mathrm{GHz}$ frequency band," in Proc. Eur. Conf. Antennas Propag., 2011, pp. $758-762$.

[3] J. R. Costa, E. B. Lima, and C. A. Fernandes, "Compact beam-steerable lens antenna for $60-\mathrm{GHz}$ wireless communications," IEEE Trans. Antennas Propag., vol. 57, no. 10, pp. 2926-2933, Oct. 2009.

[4] I. Kim and Y. Rahmat-Samii, "Beam-tilted dipole EBG array antenna for future base station applications," in Proc. IEEE Antennas Propag. Soc. Int. Symp., 2013, pp. 1224-1225.

[5] Y. Kashino, K. Sakakibara, Y. Tanaka, N. Kikuma, and H. Hirayama, "Design of millimeter-wave microstrip comb-line antenna array beamtilting in perpendicular plane of feeding line," in Proc. Asia-Pacific Microw. Conf., Dec. 2006, pp. 817-820.

[6] A. Dadgarpour, B. Zarghooni, B. S. Virdee, and T. A. Denidni, "Beam tilting antenna using integrated metamaterial loading," IEEE Trans. Antennas Propag., vol. 62, no. 5, pp. 2874-2879, May 2014.

[7] Z. Szabó, P. G. Ho, R. Hedge, and E. P. Li, "A unique extraction of metamaterial parameters based on Kramers-Kronig relationship," IEEE Trans. Microw. Theory Tech., vol. 58, no. 10, pp. 2646-2653, Oct. 2010 